\title{
THE ISM FROM THE SOFT X-RAY BACKGROUND PERSPECTIVE
}

\author{
S. L. Snowden \\ NASA/Goddard Space Flight Center \\ Universities Space Research Association \\ snowden@riva.gsfc.nasa.gov
}

\begin{abstract}
In the past few years progress in understanding the local and Galactic ISM in terms of the diffuse $X$-ray background has been as much about what hasn't been seen as it has been about detections. High resolution spectra of the local SXRB have been observed, but are inconsistent with current thermal emission models. An excess over the extrapolation of the high-energy (most clearly visible at $E>$ $1.5 \mathrm{keV}$ ) extragalactic power law down to $\frac{3}{4} \mathrm{keV}$ has been observed but only at the level consistent with cosmological models, implying the absence of at least a bright hot Galactic halo. A very recent FUSE result indicates that O VI emission from the Local Hot Bubble is insignificant, if it exists at all, a result which is also inconsistent with current thermal emission models. A short review of the current status of our (well, at least my) understanding of the Galactic SXRB and ISM is presented here.
\end{abstract}

\section{Introduction}

What is the soft X-ray diffuse background (SXRB), at least in relevance to the Galactic interstellar medium? Phenomenologically, the SXRB is whatever is left over after all other distinct identified emission sources have been removed. Bright point sources (or effective point sources) such as AGN, binary systems, black holes, and pulsars are first excised from the data. Next, bright extended structures such as clusters of galaxies and young supernova remnants which stand out from the background as distinct individual objects are removed, objects such as the Virgo Cluster, Cygnus Loop supernova remnant (SNR), and the Vela SNR. This far the winnowing process is simple with the only complication being determining the angular extent and brightness at which an object becomes a "bright extended structure," and so is removed. The next step is to account for unresolved objects which are contributing to the residual background, primarily stars and AGN, which is where the situation does get complicated. 


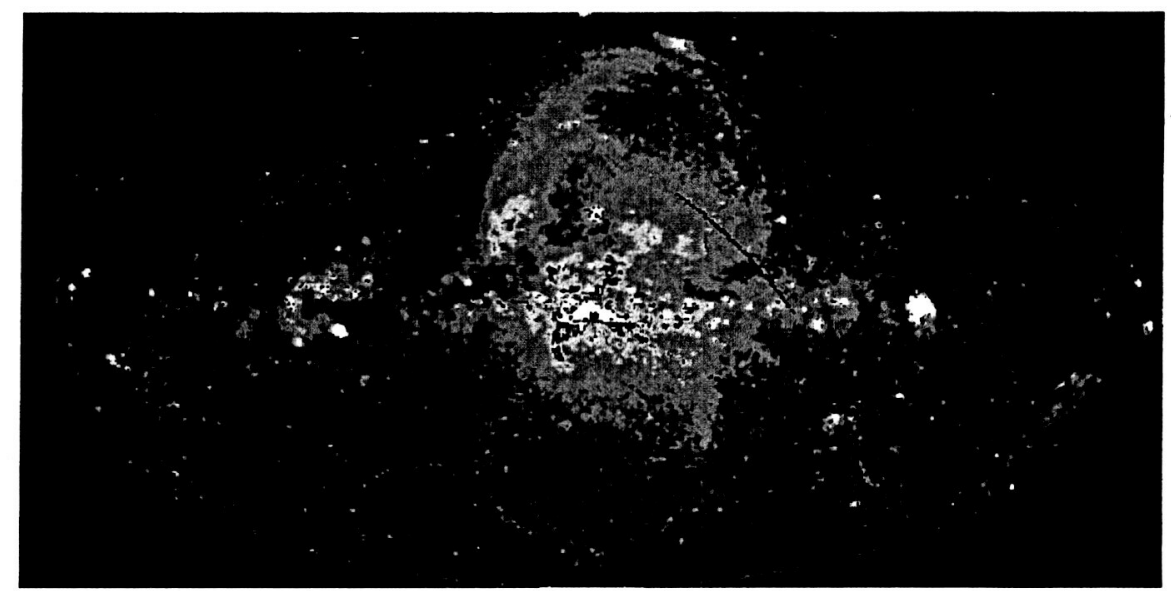

Figure 1. ROSAT All-Sky Survey (RASS) image of the $1-2 \mathrm{keV}$ band SXRB (Snowden et al. 1997), displayed with an Aitoff-Hammer zero-centered projection in Galactic coordinates. The data have been square-root scaled, longitude increases to the left, and red and white indicate brighter emission. Note the absorption minimum along the Galactic plane, the Loop I and Galactic bulge emission in the direction of the Galactic center and rising to high latitudes. The North Polar Spur (NPS) is the limb-brightened edge of the Loop I emission running diagonally north and then west from near the plane at $l \sim 30^{\circ}$ to $l, b \sim 300^{\circ}, 70^{\circ}$. Several bright but smaller objects are visible along the Galactic plane, e.g., the Cygnus Superbubble at $l \sim 90^{\circ}$ and the Vela SNR at $l \sim 270^{\circ}$. The Coma cluster is the small enhancement at the very top of the image while the Virgo cluster is the larger enhancement at the top of the NPS. The Large Magellanic Cloud is the emission region at $l, b \sim 270^{\circ},-30^{\circ}$.

The residual background component made up of cosmological objects (primarily AGN) is most clearly observed at energies above $1.5 \mathrm{keV}$ where Galactic emission is minimal. Except for absorption modulation by material of the Galactic disk it appears isotropic (Figure 1). There are several groups which have worked rather successfully on resolving this background by the use of deep surveys (e.g., Hasinger et al. 1993; Mushotzky et al. 2000; Giacconi et al. 2001). This is convenient for those of us studying the Galactic SXRB as they provide highly accurate models for what must be subtracted to leave the truly interesting signal. Unresolved Galactic stars also provide a background component but fortunately at a relatively low level which primarily affects the $\frac{3}{4} \mathrm{keV}$ band (Kuntz and Snowden 2001b).

The observed Galactic SXRB covers the energy range from about $0.1 \mathrm{keV}$ to $1.5 \mathrm{keV}$ and originates primarily as diffuse emission from thermal processes with temperatures of $k T \sim 0.1-1 \mathrm{keV}$. The lower limit of the energy band is set by technical reasons (typical detectors for observing the SXRB have very small responses approaching $0.1 \mathrm{keV}$ ) and by definition (the X-ray and EUV bands meet at about $100 \mathrm{eV}$ ). The upper limit is more nebulous being set by the 
emission mechanisms, however even the higher temperature Galactic emission is still less than $k T \sim 1 \mathrm{keV}$.

\section{A Short Guided Tour of the SXRB}

Away from the Galactic center quadrant the SXRB in the $1-2 \mathrm{keV}$ band (Figure 1) is relatively isotropic illustrating the contaminating contribution of unresolved (and therefore unremoved) extragalactic sources. Emission from the Galactic bulge and Loop I (and most clearly the NPS) dominate the Galactic center region. The Cygnus Superbubble at $l \sim 90^{\circ}$ and the Vela SNR at $l \sim 270^{\circ}$ are clearly seen along the Galactic plane. The effect of Galactic absorption is seen most clearly in the plane between $l \sim 90^{\circ}$ and $l \sim 180^{\circ}$.

Similar to the $1-2 \mathrm{keV}$ band, the $\frac{3}{4} \mathrm{keV}$ band is dominated by the unresolved extragalactic background except in the Galactic center quadrant and along the Galactic plane. However, there are a few Galactic objects which become more visible (e.g., the Eridanus Superbubble and Monoceros/Gemini SNR, the latter also known as the Monogem Ring) as the energy range of the band becomes more compatible with the emission temperatures. Galactic absorption features also become clearer as the disk becomes optically thick at higher latitudes (ISM absorption cross sections go roughly as $E^{-\frac{8}{3}}$ ).

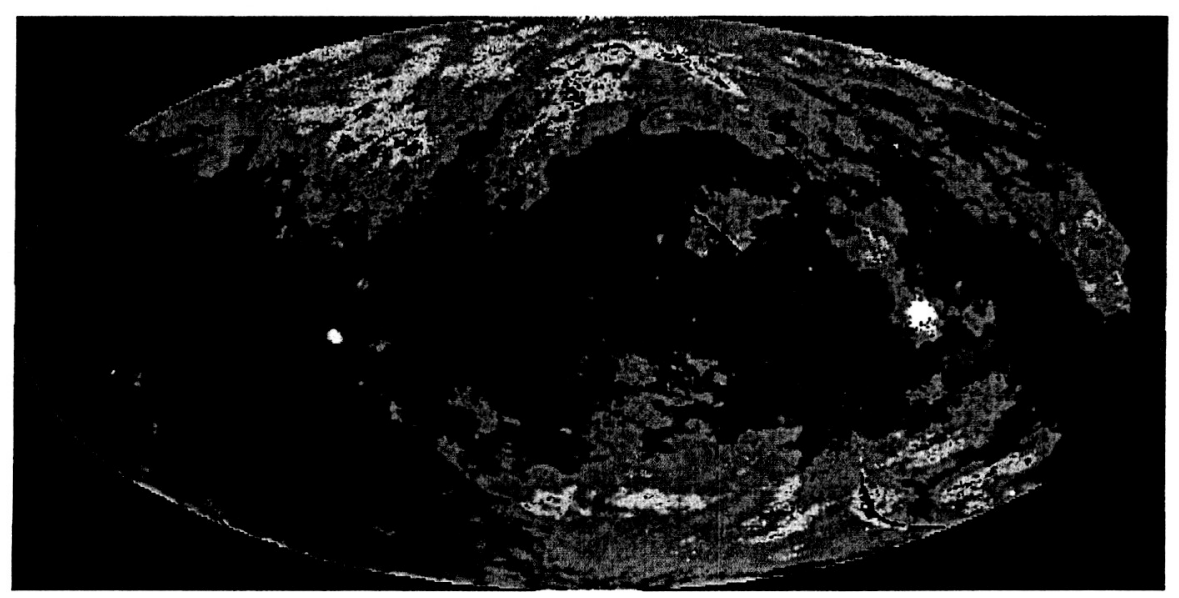

Figure 2. Same as Figure 1 except for the $\frac{1}{4} \mathrm{keV}$ band and the data have been linearly scaled. Galactic absorption is optically thick to much higher latitudes $\left(|b| \sim 30^{\circ}\right)$ and obscures all but the nearest objects in the Galactic plane. However, a number of Galactic features have become readily apparent, e.g., the Monoceros/Gemini SNR at $l, b \sim 200^{\circ}, 10^{\circ}$, the Eridanus (Eridion) Superbubble at $l, b \sim 190^{\circ},-30^{\circ}$, and the Cygnus Loop SNR at $l, b \sim 90^{\circ},-5^{\circ}$. The more northerly part of the NPS is still visible but the southern end is obscured by absorption.

The structure of the $\frac{1}{4} \mathrm{keV}$ background (Figure 2) is completely different from the higher energy bands due both to additional Galactic emission and the 
stronger effect of Galactic absorption. This is where the subject of this paper becomes critical for the goals of this conference. The Galactic $\frac{1}{4} \mathrm{keV}$ background is much brighter than the extrapolation of the isotropic extragalactic background, can and does vary significantly on angular scales of a degree or less, and of perhaps more importance for the subject of this conferences can play merry havoc with observations of soft X-ray emission from clusters of Galaxies.
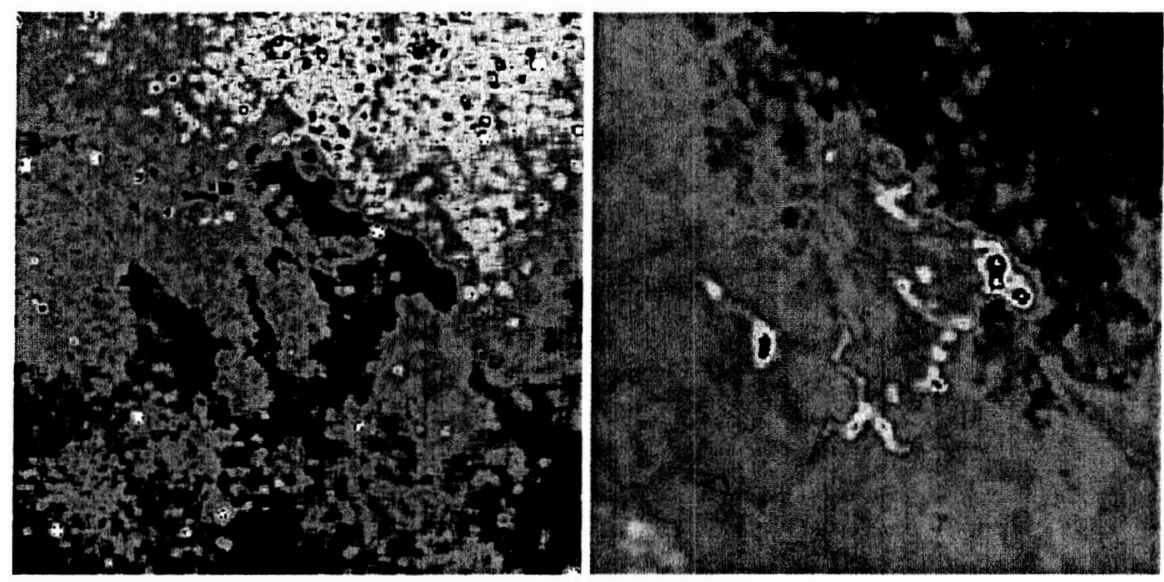

Figure 3. RASS $\frac{1}{4} \mathrm{keV}$ (left) and IRAS $100 \mu \mathrm{m}$ (right) images of the Draco Nebula. The fields are the same and are $9.0^{\circ} \times 9.0^{\circ}$ in extent. Galactic north is up and red and white indicate higher intensity. The bright knots in the X-ray data are point sources of various types. Note the detailed and fine structure of the X-ray data.

The Draco Nebula provides an exceptional example of the possible structure of the Galactic diffuse X-ray background at $\frac{1}{4} \mathrm{keV}$, and the coupling between the ISM and that structure. Figure 3 shows both the $\frac{1}{4} \mathrm{keV}$ ROSAT All-Sky Survey (RASS) and cleaned IRAS $100 \mu \mathrm{m}$ (Schlegel, Finkbeiner, and Davis 1998) data for the Draco region where the detailed negative correlation between the two data sets is striking. The negative correlation also illustrates the primary tool for locating the emission regions in space by use of the "shadowing" technique. By looking at the detailed negative correlation, i.e., the variation of SXRB intensity with absorbing column density, it is possible to model the foreground and background (to the absorbing ISM) X-ray emission. With the knowledge of the distance to the absorbing material it is possible to locate the emission in three-dimensional space. For the case of the Draco Nebula, the distance and latitude places it at the upper edge of the Galactic disk implying the existence of strong $\frac{1}{4} \mathrm{keV}$ emission in the Galactic halo, as well as significant emission in the nearest few hundred parsecs. 


\section{The Hot Phase of the Galactic ISM}

Now for the questions: What is the hot phase of the Galactic ISM? Where is it located? How much of it is there? Where did it come from?

What is it? The hot ISM is the remnant of various energetic processes in the Galaxy such as supernovae and stellar winds. The X-ray emitting plasmas are diffusely distributed over volumes extending hundreds of parsecs, and in the case of the Galactic bulge several kiloparsecs. The plasmas have temperatures of $k T \sim 0.1-1 \mathrm{keV}$ and correspondingly low space densities $\left(n_{e} \sim 0.01 \mathrm{~cm}^{-2}\right)$. Recent observations have confirmed that at least the $\frac{1}{4} \mathrm{keV}$ emission is thermal in nature and dominated by various lines (Sanders et al. 2001). The observations also showed that the emission is inconsistent with that from a plasma in thermal equilibrium with solar abundances.

Where is the $\frac{1}{4} \mathrm{keV}$ emission? Making use of the RASS and cleaned IRAS $100 \mu \mathrm{m}$ data in a shadowing analysis it has been possible to produce a picture of at least the local (out to a few hundred parsecs in the plane, farther in the halo) $\frac{1}{4} \mathrm{keV}$ emission (Snowden et al. 1998). From ISM optical absorption line studies the Sun is located in a cavity in the $\mathrm{H}$ I of the Galactic disk extending from less than $\sim 50 \mathrm{pc}$ from the Sun in the plane to well over $100 \mathrm{pc}$ at high Galactic latitudes (e.g., Sfeir et al. 1999). From the X-ray data it is clear that this cavity contains an extensive distribution of thermal plasma at $k T \sim 0.1 \mathrm{keV}$, which has been given the name the Local Hot Bubble (LHB). A few distinct emission regions such as the Monoceros/Gemini SNR, Eridanus Superbubble, Vela SNR, and Cygnus Loop SNR mentioned above can be seen near the plane out to several hundred parsecs, but that is about the limit as ISM absorption cuts off observations of emission regions at greater distances. At higher Galactic latitudes absorption optical depths for $\frac{1}{4} \mathrm{keV} \mathrm{X}$-rays drop significantly to a minimum of $\tau \sim 0.5$ but with typical values from $\sim 1-1.5$. The $\mathrm{X}$-ray emission in the halo appears rather patchy with intensities ranging from near zero to many times typical values for the LHB.

Where is the $\frac{3}{4} \mathrm{keV}$ emission? The two largest and brightest $\frac{3}{4} \mathrm{keV}$ Galactic emission regions as seen from the Earth unfortunately lie in the same direction, that of the Galactic center. The Loop I superbubble is powered by the Sco-Cen $\mathrm{OB}$ associations and has a radius of $\sim 100 \mathrm{pc}$ and is centered at a distance of $\sim 150 \mathrm{pc}$ in the direction of $l, b \sim 330^{\circ}, 15^{\circ}$. The Galactic bulge is most clearly seen in the southern hemisphere where it is not confused with the Loop I emission. It appears to have a radial extent of $\sim 5 \mathrm{kpc}$, a scale height of $\sim 2 \mathrm{kpc}$, a temperature of $k T \sim 0.4-0.5 \mathrm{keV}$, and a luminosity of $\sim 2 \times 10^{39} \mathrm{ergs} \mathrm{s}^{-1}$ (Snowden et al. 1997). It is not clear whether there exists a large scale-height $\frac{3}{4} \mathrm{keV}$ halo such as suggested by the work of Wang (1998). Kuntz and Snowden (2001a) and Kuntz, Snowden, and Mushotzky (2001) place restrictive limits on the amount of halo and truly extragalactic 
emission in this band. Any such extended halo emission is going to be confused with more cosmological emission, at least to the extent of the Local Group of galaxies. A recent high-resolution spectrum of the diffuse background places strong limits on the spectral distribution of the $\frac{3}{4} \mathrm{keV}$ background based on the $O$ VII and $O$ VIII emission lines (McCammon et al. 2002). The oxygen results when combined with the deep survey AGN spectra show that $42 \%$ of the observed flux can be attributed to unresolved AGNs and a minimum of $36 \%$ to thermal emission at zero redshift (which would include the contributions of both unresolved stars and the cooler plasma contributing to the $\frac{1}{4} \mathrm{keV}$ background). Subtracting the high-latitude contribution of unresolved stars, LHB, and cooler halo leaves an upper limit of $\sim 20 \%$ of the observed flux which (because of its low redshift) has either a Galactic halo or local group origin.

How much of it is there? The emission at $\frac{3}{4} \mathrm{keV}$ from the Galactic bulge is well constrained, as is the hotter Galactic halo, at least by upper limits (see above). At $\frac{1}{4} \mathrm{keV}$ the amount of emission from the LHB is fairly well constrained as is the nearest few hundred parsecs to kiloparsec of the Galactic halo. However, the amount of $k T \sim 0.1 \mathrm{keV}$ plasma in the disk of the Milky Way as a whole is essentially unknown as it is invisible to us. The fact that the $\frac{1}{4} \mathrm{keV}$ halo appears so patchy suggests that it lies at a relatively low scale height and presumably just above the neutral gas of the disk. If this is the case then we know little more about the extent of the cooler halo as we can only sample $\sim 1 \%$ of the Galaxy.

Where did it come from? The bottom line is that the production of extensive plasma with temperatures in the $k T \sim 0.1-1 \mathrm{keV}$ range requires very energetic phenomena such as supernovae and the stellar winds of OB associations and star forming regions. This is clearly the case for many of the distinct objects mentioned above such at the Loop I superbubble powered by the ScoCen $O B$ associations and the Eridanus Superbubble powered by the Orion OB associations. The Galactic bulge and the hotter part of the Galactic halo (if it exists) may be powered by star formation and perhaps infall from the IGM. The cooler part of the Galactic halo can be produced either by the breakout of emission regions in the disk (otherwise known as Galactic fountains) or in-situ by halo supernovae. The LHB appears to be an aging supernova remnant with no candidate for the originating object. The physical extent of the local cavity suggests that in was created through the effort of more than one supernova, and plasmas at $k T \sim 0.1 \mathrm{keV}$ are relatively long lived with cooling times of millions of years providing a time scale where that would not be unreasonable. Małz-Apellaniz (2001) suggests that a component of the Sco-Oph OB associations passed through the region of the local cavity several million years ago and a supernova at that time could have reheated the plasma in an existing cavity. The occurrence of a supernova within the last five million years and within 
$30 \mathrm{pc}$ of the Sun is supported by the results of Knie et al. (1999) who analyzed

${ }^{60} \mathrm{Fe}$ (a supernova byproduct) in ocean-floor sediment.

\section{Caveats and Other Considerations}

The McKee and Ostriker model for the ISM: I raise this subject as there seemed to be some surprise at the conference that although the model had a good run it has been effectively ruled out. McKee and Ostriker (1977) postulated an ISM comprised of a highly clumped cooler component ( $\mathrm{H}$ I clouds) embedded in a hotter matrix (X-ray emitting plasma) where the $\mathrm{O}$ VI observed by Copernicus could arise from the interface region between the two. This model solved a number of model/data inconsistencies at the time and proved rather popular over the years. However, such a clumped distribution of cold clouds has been searched for but has not been observed over all reasonable angular scales in $21-\mathrm{cm}$ emission and absorption. In addition, the X-ray data do not show any support for the interconnecting matrix of hot plasma. (See Snowden 2001 for a somewhat more extended discussion of this subject).
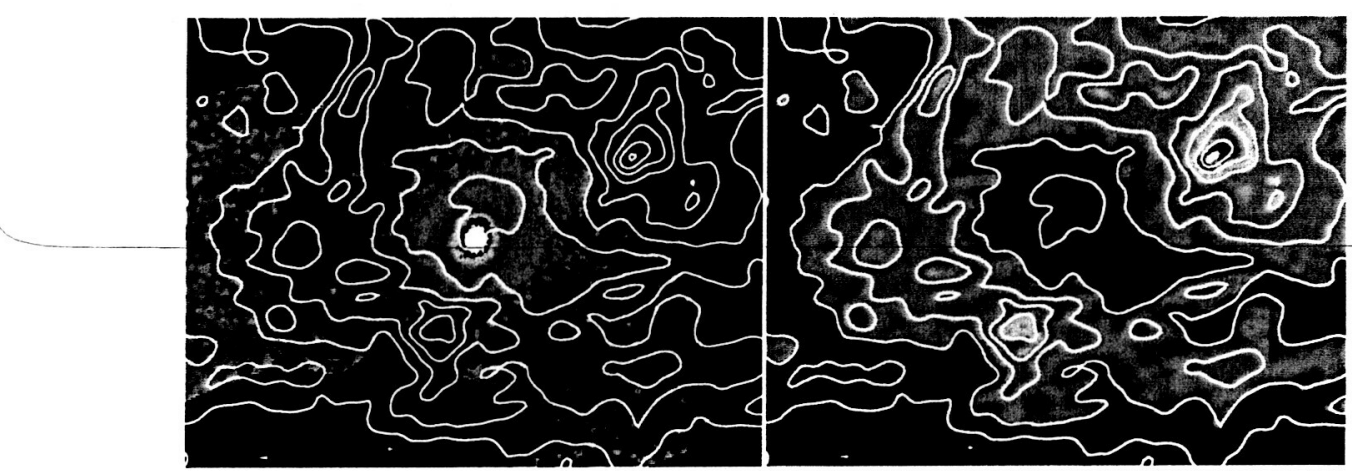

Figure 4. ROSAT $\frac{1}{4} \mathrm{keV}$ (left) and IRAS $100 \mu \mathrm{m}$ (right) images of the Virgo Cluster. The fields are the same and are $\sim 6^{\circ} \times 5^{\circ}$ in extent. Red and white indicate higher intensity. The peak IRAS $100 \mu \mathrm{m}$ intensities correspond to several optical depths at $\frac{1}{4} \mathrm{keV}$.

The pathological nature of the Virgo Cluster region: This digression is included as a cautionary tale about the analysis of soft X-ray data and the effects of ISM absorption. Figure 4 shows the $\frac{1}{4} \mathrm{keV}$ and IRAS $100 \mu \mathrm{m}$ images of the Virgo Cluster region, the most pathological case I know of where ISM absorption conspires to confuse the analysis of a completely unrelated object. The ring of IRAS cirrus surrounds the brightest region of the cluster emission with $\sim 1-2$ optical depths of additional absorption at $\frac{1}{4} \mathrm{keV}$. Because the cirrus ring is roughly circular as well as roughly centered on the cluster it significantly modifies the radial profile of the $\frac{1}{4} \mathrm{keV}$ emission. However, the 
additional absorption is relatively small at higher energies so the radial profile of, for instance, the $\frac{3}{4} \mathrm{keV}$ to $\frac{1}{4} \mathrm{keV}$ hardness ratio is also affected.

Where has all the $O$ VI from the LHB gone? Shelton (2003) presents results which throw some confusion on models for the LHB and cold cloud/hot plasma interfaces. Using FUSE observations there appears to be little or no $\mathrm{O}$ VI emission from the LHB where there should be $T \sim 300,000 \mathrm{~K}$ gas in interface regions between the hotter plasma and the cooler material of the cavity walls. There should also be O VI emission from the small clouds located within the cavity (the Sun itself is located in the "Local Fluff," a region of partially ionized gas at a few thousand degrees with an extent of a few parsecs).

Current spectral models don't fit the observed spectra: While there are a few relatively good spectra of the diffuse background at $\frac{1}{4} \mathrm{keV}$ they are not well fit by current thermal equilibrium and normal abundance emission models, or non-equilibrium models for that matter (Sanders et al. 2001).

What is the zero level of the $\frac{1}{4} \mathrm{keV} X$-ray data? Recently the production of soft X-rays in the solar system by charge exchange between the solar wind and interstellar neutrals has become a hot topic. While so far this mechanism has been used to explain the flaring long-term enhancement background observed in the RASS (Cravens, Robertson, and Snowden 2001), the question arises whether at quiesence it still produces a non-negligable flux which could significantly impact our view of the LHB (e.g., Lallement 2003).

\section{References}

Cravens, T. E., Robertson, I. P., and Snowden, S. L. 2001, JGR, 106, 24883

Giacconi, R., et al. 2001, ApJ, 551, 624

Hasinger, G., et al. 1993, A\&A, 275, 1

Knie, K., et al. 1999, PhRvL, 83, 18

Kuntz, K. D., and Snowden, S. L. 2001a, ApJ, 543, 195

Kuntz, K. D., and Snowden, S. L. 2001b, ApJ, 554, 684

Kuntz, K. D., Snowden, S. L., and Mushotzky, R. F. 2001, ApJL, 548, 119

Lallement, R. 2003, A\&A, submitted

Małz-Apellaniz, J. 2001, ApJL, 560, L83

McCammon, D., et al. 2002, ApJ, 576, 188

McKee, C. F., and Ostriker, J. P. 1977, ApJ, 218, 148

Mushotzky, R. F., Cowie, L. L., Barger, A. J., and Arnaud, K. A. 2000, Nature, 404, 459

Sanders, W. T., et al. 2001, ApJ, 554, 694

Schlegel, D. J., Finkbeiner, D. P., and Davis, M. 1998, ApJ, 500, 525

Sfeir, D. M., Lallement, R., Crifo, F., and Welsh, B. Y. 1999, A\&A, 346, 785

Shelton, R. 2003, ApJ, in press

Snowden, S. L. 2001, in The Century of Space Science, Klewer Academic Publishers, 581

Snowden, S. L., et al. 1997, ApJ, 485, 125

Snowden, S. L., et al. 1998, ApJ, 493, 715

Wang, Q. D. 1998, Lecture Notes in Physics, 506, 503 